\title{
Collective excitations in quasi-one-dimensional electron systems under a magnetic field
}

\author{
B. Tanatar \\ Department of Physics, Bilkent University, Bilkent, 06533 Ankara, Turkey \\ N. C. Constantinou \\ Department of Physics, University of Essex, Colchester $\mathrm{CO}_{4} 3 \mathrm{SQ}$, England
}

(Received 15 July 1993)

\begin{abstract}
A study of the magnetoplasmons of a cylindrical quasi-one-dimensional electron system is given in the presence of an axial magnetic field $B$. A two-subband system is considered and the dispersion relations for both intrasubband and intersubband magnetoplasmons are obtained using the exact infinite wall eigenfunctions. It is demonstrated that the application of a longitudinal $B$ changes the mode frequencies for both types of excitations. The model includes both local-field corrections, which are shown to be important, and the Hartree potential, which is given in closed form.
\end{abstract}

Recent developments in the fabrication techniques such as molecular-beam epitaxy and lithographic deposition have made possible the realization of quasi-onedimensional (Q1D) electron systems. In these structures based on the confinement of electrons, the electron gas is quantized in two transverse directions, thus the charge carriers essentially move only in the longitudinal direction. Collective excitations of a Q1D electron system, both in the absence and presence of an external magnetic field, are subject to growing experimental ${ }^{1-6}$ and theoretical $^{7-11}$ interest. Using magnetic depopulation, ${ }^{5}$ far-infrared ${ }^{1}$ and Raman spectroscopy ${ }^{3}$ techniques, plasmon dispersion in GaAs quantum wires is measured.

Recently, $\mathrm{Hu}$ and Das Sarma ${ }^{12}$ have shown convincingly the reason one-dimensional (1D) quantum wire electrons behave as normal Fermi liquids, despite the theoretical claims of the existence of non-Fermi-liquid-type ground states (i.e., Luttinger liquid). They have demonstrated, within the random-phase approximation (RPA), that particles at the Fermi surface can excite low-energy virtual plasmons. A theoretical account of the elementary excitation spectrum of Q1D electron systems under magnetic fields, within a parabolic confining potential, was given by $\mathrm{Li}$ and Das Sarma. ${ }^{9}$ They have studied the magnetoplasmon modes using the RPA and a perturbation expansion method and investigated the effects of $B$. The main difference between our work and that of $\mathrm{Li}$ and Das Sarma ${ }^{9}$ is that the applied field is transverse in their case to the free direction, hence motion in this direction is affected, whereas in our model the $B$ lies along the free direction.

Owing to the limited number of available final states during the scattering process, the mobility of Q1D electron systems are considerably enhanced, making them potentially important for high-speed device applications. Since the early prediction by Sakaki ${ }^{13}$ and subsequent fabrication, ${ }^{6}$ there has been much interest in the transport properties of Q1D systems. The hope of achieving very high mobilities by confining even more electrons compared with the Q2D heterojunctions has been the main motivation of the study of Q1D electron systems.

Our primary aim in this paper is to study the collective excitation spectrum (magnetoplasmon modes) of a Q1D electron system under a magnetic field (in the axial direction) using the quantum-well potential model developed recently by Constantinou, Masale, and Tilley. ${ }^{14}$ We consider a two-subband model for the system and employ the RPA to investigate the intrasubband and intersubband magnetoplasmons.

We consider a model of the electron gas, quantized in two transverse directions, so that the charge carriers can only move in the longitudinal direction. We choose the cross section of the system to be circular with radius $R$, hence the quantum wire geometry becomes cylindrical. The external magnetic field is applied parallel to the longitudinal axis. In the size quantum limit, the radius $R$ of the quantum-well wire is much smaller than the thermal de Broglie wave length of the charge carriers, so that only the lowest subband is populated. The key parameter in the description of the motion of an electron in a magnetic field is the ratio $l_{B} / R$, where $l_{B}^{2}=\hbar c / e B$ is the cyclotron radius. For $l_{B} \ll R$, the electron is strongly confined by the magnetic field, whereas in the opposite limit $l_{B} \gg R$ the confinement due to $B$ becomes unimportant. ${ }^{14}$ In the model of an infinite potential well confining the charge carriers, the effective-mass wave function is given by ${ }^{14,15}$

$$
\psi \sim \exp (-\xi / 2) \xi^{|m| / 2} M(a, b, \xi),
$$

where $\xi=r^{2} / 2 l_{B}^{2}$ is a dimensionless variable and $M(a, b, \xi)$ is the confluent hypergeometric function with the arguments defined as $a=-E_{m l} / \hbar \omega_{c}+\frac{1}{2}(1+m+|m|)$ and $b=|m|+1$. The eigenvalues $E_{m l}$ are obtained by solving $M\left(a, b, R^{2} / 2 l_{B}^{2}\right)=0$, with the index $l$ denoting the $l$ th root.

The Coulomb interaction matrix elements between the subbands is given by

$$
\begin{aligned}
V_{i j k l}(q)=\frac{2 e^{2}}{\kappa} \int d^{2} r \int & d^{2} r^{\prime} \psi_{i}(r) \psi_{j}(r) K_{0}\left(q\left|\mathbf{r}-\mathbf{r}^{\prime}\right|\right) \\
& \times \psi_{k}\left(r^{\prime}\right) \psi_{l}\left(r^{\prime}\right)
\end{aligned}
$$

where $K_{0}(x)$ is the modified Bessel function of the second kind, and $\kappa$ is the background dielectric constant. In the following we specialize to a two-subband system in which the lower level described by $m=0$ is completely filled and the upper level described by $m=-1$ is empty. 
Such a model will have relevance, since it is possible to fabricate Q1D electron systems in the form of quantumwires under similar conditions. ${ }^{6}$

We define, employing the RPA, the dielectric tensor of a multisubband system by

$$
\varepsilon_{i j m n}(q, \Omega)=\delta_{i j} \delta_{m n}-V_{i j m n}(q) \Pi_{i j}(q, \Omega),
$$

in which $\Pi_{i j}(q, \Omega)$ is the generalized susceptibility for which the lowest order approximation is provided by the Lindhard function

$$
\Pi_{i j}(q, \Omega)=2 \sum_{k} \frac{f\left(E_{i, k+q}\right)-f\left(E_{j, k}\right)}{\hbar \Omega+E_{i, k+q}-E_{j, k}+i \eta},
$$

where $f(x)$ is the Fermi distribution function and $\eta$ is a positive infinitesimal quantity. The collective modes for our two-subband model of a quantum-well wire may be obtained from

$$
\left[1-V_{1111}(q) \Pi_{11}(q, \Omega)\right]\left[1-V_{1221}(q) \Pi_{12}(q, \Omega)\right]=0 .
$$

The first collective mode, which is the intrasubband magnetoplasmon, involving the motion of electrons in the free direction, is given by

$$
1-V_{1111}(q) \Pi_{11}(q, \Omega)=0
$$

whereas the second mode, the intersubband magnetoplasmon, associated with the transitions between the subbands is obtained from the solution of

$$
1-V_{1221} \Pi_{12}(q, \Omega)=0 .
$$

Using the long-wavelength limit, viz. $q \rightarrow 0$, of $\Pi_{11}$ and $\Pi_{12}$, we obtain for the intrasubband magnetoplasmon dispersion relation

$$
\left(\frac{\Omega_{q}}{\epsilon_{F}}\right)^{2}=\frac{32 r_{s}}{\pi^{2} \kappa} q^{2} f(q)\left[1+\left(1+\frac{\pi^{2} \kappa f_{1}(q)}{16 r_{s} q^{2} f(q)}\right)^{1 / 2}\right],
$$

where $r_{s}=4 / \pi k_{F} a_{B}$ is the $1 \mathrm{D}$ electron gas parameter expressed in terms of the Bohr radius $a_{B}, f(q)$ is defined through $V_{i j m n}=2 e^{2} / \kappa f(q)$, and $f_{1}(q)=q^{2}\left(q^{2}+4\right)$. The intersubband magnetoplasmon dispersion is obtained as

$$
\left(\Omega_{q} / \epsilon_{F}\right)^{2}=\left[A(q) \omega_{+}^{2}-\omega_{-}^{2}\right] /[A(q)-1],
$$

where $A(q)=\exp \left\{\frac{\pi^{2} \kappa}{8 r_{s}} \frac{q}{f(q)}\right\}$ and $\omega_{ \pm}=E_{21} / \epsilon_{F} \pm 2 q+q^{2}$, with $E_{21}=E_{2}-E_{1}$ being the difference between the subband energy levels. In the above equations the wave vector $q$ is in units of the Fermi wave vector $k_{F}$.

We use parameters appropriate for GaAs in the numerical calculations. The electron effective mass is $m=$ $0.067 m_{e}$, where $m_{e}$ is the bare electron mass, and the dielectric constant is $\kappa=12.9$. In Fig. 1 , we illustrate the magnetic field dependence of the eigenvalue spectrum of a cylindrical quantum-well wire of radius $R=300 \AA$ (see also Ref. 14). For the present model we restrict our calculations to the two lowest subbands, labeled $m=0$ and -1 and indicated by solid lines. Also shown (by dotted lines) are some of the higher subbands. Clearly, for $B \geq 15 \mathrm{~T}$ the subband energies are rather close, and we do not expect a strong $B$ dependence of the collective

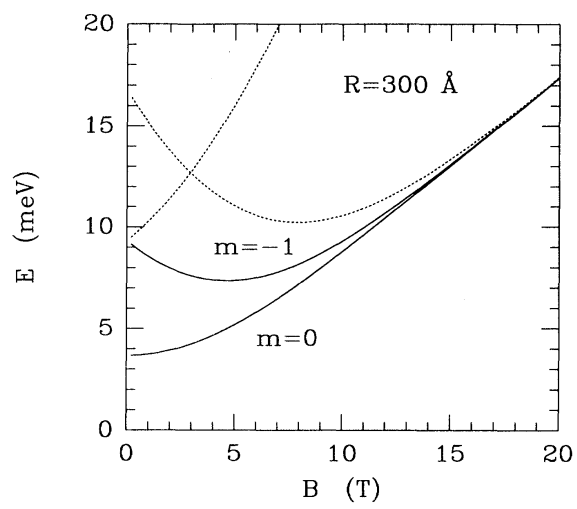

FIG. 1. The lowest order subband energies as a function of magnetic field for an infinite potential quantum-well wire. The cylindrical wire radius is taken to be $R=300 \AA$. The two lowest subbands are shown by the solid lines and higher subbands are indicated by the dotted lines.

excitations beyond this point. When the wire radius is less than $\sim 100 \AA$ the magnetic field dependence of the subband energies are rather weak. ${ }^{14}$ Thus to obtain the enhanced effects of the external field $B$, we choose to work mostly with $R=300 \AA$ and the electron number density $N=0.2 \times 10^{6} \mathrm{~cm}^{-1}$ in the rest of the paper.

The intrasubband magnetoplasmon dispersion curves for various magnetic fields are shown in Fig.1(a). The solid lines indicate the boundary of the particle-hole continuum. The dotted, long-dashed, and short-dashed lines are for $B=0,1$, and $10 \mathrm{~T}$, respectively. We note that for the electron density $N=0.2 \times 10^{6} \mathrm{~cm}^{-1}$ and wire radius $R=300 \AA$, the intrasubband magnetoplasmons enter the particle-hole continuum at some magnetic field dependent $q_{c}$. We obtain $q_{c} \approx 1.55,1.65$, and $2 k_{F}$ for $B=0$, 1 , and $10 \mathrm{~T}$, respectively. In general, the magnetic field increases $q_{c}$, the wave vector at which the plasmon mode enters the single particle continuum. This Landau damping of the magnetoplasmon modes are absent at smaller wire radii. In fact, in different forms of the transverse wave function $^{16,17}$ of the Q1D electron systems, the Landau damping of the plasmons (at zero-field) was not seen. Figure 2(b) shows the intersubband magnetoplasmon excitation energies for $N=0.2 \times 10^{6} \mathrm{~cm}^{-1}$ and $R=300 \AA$. The dotted, long-dashed, and short-dashed lines are for $B=0,1$, and $10 \mathrm{~T}$, respectively. Note that the intersubband excitation energies for the collective modes do not have a monotone $B$ dependence. The excitation energies for $B=1 \mathrm{~T}$ are about twice that for $B=0$, but the energies for $B=10 \mathrm{~T}$ actually are lower than the zerofield case. The intersubband magnetoplasmon energies show a minimum as a function of $q$ similar to the results obtained by $\mathrm{Li}$ and Das Sarma. ${ }^{9}$ As the wire radius is decreased, this behavior is less pronounced.

We display in Fig. 3 the dependence of intrasubband excitation energy as a function of the wire radius $R$ for $N=0.2 \times 10^{6} \mathrm{~cm}^{-1}$ and $q=k_{F}$. The dotted, longdashed, and short-dashed lines refer to $B=0,1$, and $10 \mathrm{~T}$, respectively. Here, for each value of the magnetic field $B$, we first determine corresponding energy levels by solving the eigenvalue equation and construct the full wave functions. We then calculate the magnetoplasmon 

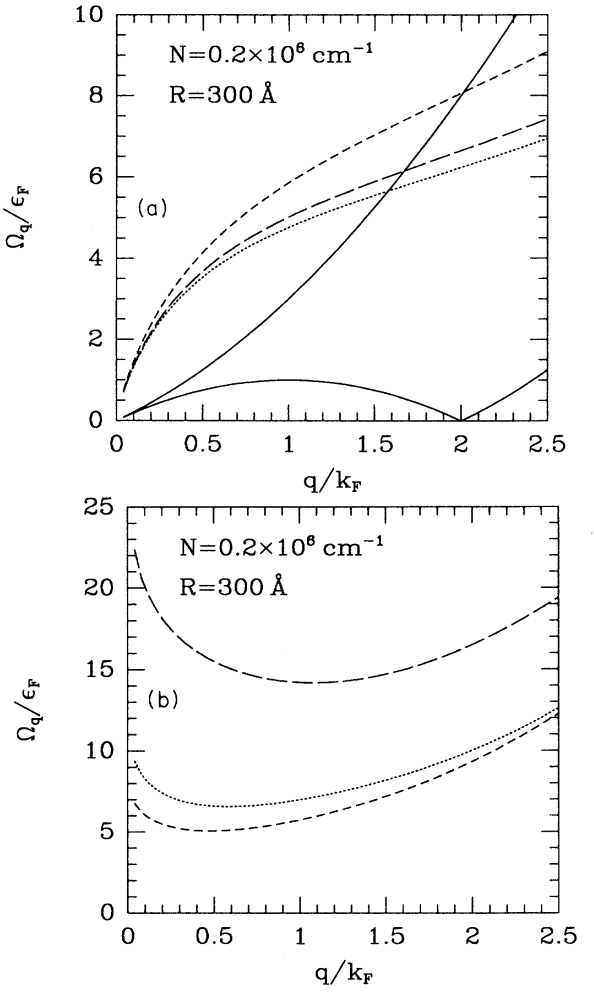

FIG. 2. (a) Intrasubband magnetoplasmon dispersion relation. Solid lines show the boundary of particle-hole continuum, dotted, long-dashed, and short-dashed lines are for $B=0,1$, and $10 \mathrm{~T}$, respectively. (b) Intersubband magnetoplasmon dispersion relation. Solid lines show the boundary of particle-hole continuum, dotted, long-dashed, and shortdashed lines are for $B=0,1$, and $10 \mathrm{~T}$, respectively.

dispersion using the full wave functions. The magnetic field dependent energy levels may be approximated by the formula given by Dingle, ${ }^{18}$

$$
\begin{aligned}
E_{m l}= & \left(\hbar^{2} / 2 m R^{2}\right) t_{m l}^{2}+\frac{1}{2} m \hbar \omega_{c} \\
& \left.+\frac{1}{24} \hbar \omega_{c}\left(R / l_{B}\right)^{2}\left\{1+\left[2\left(m^{2}-1\right)\right] / t_{m l}^{2}\right]\right\},
\end{aligned}
$$

in which $t_{m l}$ is the $l$ th root of the Bessel function $J_{m}(x)$ and $\omega_{c}$ is the cyclotron frequency. We have found that the above expression is quite good for small $B$, i.e., $B \leq$ $1 \mathrm{~T}$ and wire radii $R \leq 500 \AA$, and for $B=10 \mathrm{~T}$ and $R \leq 250 \AA$, with a maximum error of $10 \%$.

It is also possible to include the effects of the localfield corrections on the plasmon dispersion relation. The local-field effects, described by $G(q)$ [we consider a static approximation for $G(q)$ ], could be incorporated in our calculations of the plasmon dispersion relations. Evidently, one has to replace the bare Coulomb interaction $V_{i j m n}(q)$ by $V_{i j m n}(q)[1-G(q)]$. Although a selfconsistent scheme to calculate $G(q)$ may be developed along the derivation of Singwi et al. ${ }^{19}$ we take a simpler form

$$
G_{H}(q)=\frac{1}{2}\left\{\left[V_{i j m n}\left(\sqrt{q^{2}+k_{F}^{2}}\right)\right] / V_{i j m n}(q)\right\},
$$

both for intrasubband and intersubband plasmons, using
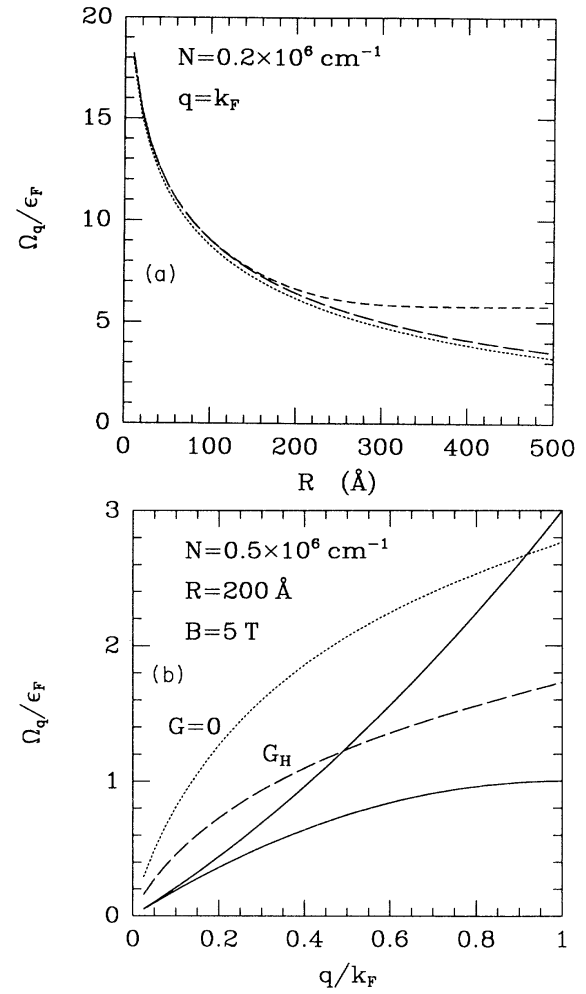

FIG. 3. (a) Intrasubband excitation energies as a function of the wire radius $R$. The dotted, long-dashed, and shortdashed lines refer to $B=0,1$, and $10 \mathrm{~T}$, respectively. (b) The effect of local-field correction $G_{H}(q)$ in the Hubbard approximation on the intrasubband magnetoplasmon dispersion. Solid lines indicate the boundary of particle-hole continuum, dotted and long-dashed lines are for $G=0$ and $G_{H}$, respectively.

$V_{1111}(q)$ and $V_{1221}(q)$, respectively. The above form of $G(q)$ amounts to the usual Hubbard's approximation to the local-field corrections. In Fig. 3(b) we show the effect of $G_{H}$ on the intrasubband magnetoplasmons. Here we take the electron number density $N=0.5 \times 10^{6} \mathrm{~cm}^{-1}$, $R=200 \AA$, and $B=5 \mathrm{~T}$. Our calculations show that the local-field corrections have a significant effect on the magnetoplasmon dispersion and perhaps a more sophisticated ${ }^{19}$ approach in the calculation of $G(q)$ is necessary. Corrections due to $G(q)$ become smaller as the electron density decreases when a finite external field is present, as noted by Gold and Ghazali ${ }^{10}$ for the zero-field case. Similar conclusions hold true for the intersubband magnetoplasmons.

We may introduce the following approximate form to the wave functions appropriate for the lowest states (viz. $l=0$ and $m=0, \pm 1, \ldots)$

$\psi \sim\left(1-r^{2} / 4 l_{B}^{2}\right)\left(r^{2} / 2 l_{B}^{2}\right)^{|m| / 2}\left(1-r^{2} / R^{2}\right) e^{i m \theta}$,

which satisfy the condition $\psi(R)=0$. The normalization constants here are different from those for the full wave functions and may be evaluated analytically. We have found that these approximate wave functions are in good agreement with the exact ones for a range of $B$ and $R$ values of practical purposes. They allow us to calculate 
the Coulomb matrix elements and magnetoplasmon dispersion relations analytically. We shall discuss results obtained using approximate wave functions elsewhere, but consider one aspect below as an example.

In our model of the Q1D electron systems, the charge carriers are confined by an infinite potential barrier. When the subbands are filled, an additional potential, which may be approximated by a Hartree term, arises. Assuming that only the lowest subband is occupied, the electron density produces a potential $\Sigma(r)$, obtained from the solution of the Poisson equation

$$
\frac{d^{2}}{d r^{2}} \Sigma(r)+\frac{1}{r} \frac{d}{d r} \Sigma(r)=-\frac{4 \pi e^{2} N}{\kappa}\left|\psi_{1}(r)\right|^{2},
$$

with the condition $\Sigma(R)=0$. The above equation for $\Sigma(r)$ may be solved numerically for the full wave function in the lowest subband to calculate the band bending, ${ }^{10}$ namely the shift in the subband energy levels due to this potential. Here we illustrate this effect, by approximating the wave function using Eq. (12), which is valid for small magnetic fields. In this case, the potential $\Sigma(r)$ becomes

$$
\begin{aligned}
\Sigma(r)= & \frac{3 e^{2} N}{\kappa} \frac{1}{\left(1-\xi_{R} / 4\right)}\left[\frac{11}{18}-\frac{29}{144} \xi_{R}-x^{2}\right. \\
& +\frac{1}{2}\left(1+\xi_{R} / 2\right) x^{4} \\
& \left.-\frac{1}{9}\left(1+2 \xi_{R}\right) x^{6}+\frac{1}{16} \xi_{R} x^{8}\right]
\end{aligned}
$$

with $\xi_{R}=R^{2} / 2 l_{B}^{2}$ and $x=r / R$. Note that the above expression reduces to the result given by Gold and Ghazali $^{10}$ in the zero-field limit. The change in the energy level $\Delta E_{1}$, due to $\Sigma$, calculated using first-order perturbation theory yields

$$
\Delta E_{1}=\left(e^{2} N / 60 \kappa\right)\left[73+\frac{172}{7} \xi_{R}+\mathcal{O}\left(\xi_{R}^{2}\right)\right],
$$

which again coincides with the results of Ref. 10 at $B=0$. We conclude from the above expressions that the intersubband energy separation will decrease with increasing electron density $N$ and also with increasing magnetic field $B$.
There are a number of possible extensions to the twosubband model of collective excitations of a quantum-well wire presented in this study. First, in view of the experimental realization of these systems, it may be more interesting to investigate the excitation spectrum of lateral quantum-well wire superlattices. Results from such structures might be more relevant when compared with experiments. Also the effects of disorder on the magnetoplasmon dispersion should be considered.

Another direction to pursue is to examine a system with more than two subbands. As discussed previously, in the present approximation intersubband and intrasubband plasmons are completely decoupled. In the treatment of Li and Das Sarma ${ }^{9}$ a three-subband system (the first two filled and the highest one empty), in which mode coupling between different type of plasmons exists, was shown to yield better agreement with data. Evidently, the collective excitation spectrum of the Q1D system gets more complex when mode coupling is allowed, for the distinction between the intrasubband and intersubband excitations becomes less clear. To render the cylindrical quantum-well wire model studied here more realistic, we may consider a finite potential well. ${ }^{14}$ Investigations $^{20}$ of a finite potential well model have shown that scattering rates are lowered by $\sim 25 \%$ in comparison to the infinite potential well model. We might expect changes, although not qualitative, in this case too.

In this paper, we have presented results for the intrasubband and intersubband magnetoplasmon excitations for Q1D structures under magnetic fields of arbitrary strength. We have also given an analytic expression for the Hartree potential $\Sigma$ in a longitudinal $B$. A more systematic study of our model along the lines of extensions and ramifications outlined above, including analytical expressions obtained using approximate wave functions, is under way and will be presented elsewhere.

B. T. gratefully acknowledges the support of this work by the Scientific and Technical Research Council of Turkey (TUBITAK) under Grant No. TBAG-1155, and fruitful discussions with Professor S. T. Chui. N.C.C. thanks the United Kingdom Science and Engineering Research Council for financial support.
${ }^{1}$ T. Demel et al., Phys. Rev. B 38, 12732 (1988).

${ }^{2}$ T. Egeler et al., Phys. Rev. Lett. 65, 1804 (1990).

${ }^{3}$ A. R. Goñi, et al. Phys. Rev. Lett. 67, 3298 (1991).

${ }^{4}$ A. S. Plaut et al., Phys. Rev. Lett. 67, 1642 (1991).

${ }^{5}$ K. F. Berggren et al., Phys. Rev. Lett. 57, 1769 (1986).

${ }^{6}$ T. J. Thornton et al., Phys. Rev. Lett. 56, 1198 (1986); J. Cibert et al., Appl. Phys. Lett. 49, 1275 (1986); H. Temkin et al., ibid. 40, 413 (1987); G. J. Iafrate et al., Surf. Sci. 113, 485 (1982).

${ }^{7}$ Q. Li and S. Das Sarma, Phys. Rev. B 40, 5860 (1989); 43, 11768 (1991).

${ }^{8}$ Q. Li and S. Das Sarma, Phys. Rev. B 41, 10268 (1990); A. Gold, Z. Phys. 89, 213 (1992).

${ }^{9}$ Q. P. Li and S. Das Sarma, Phys. Rev. B 44, 6277 (1991).

${ }^{10}$ A. Gold and A. Ghazali, Phys. Rev. B 41, 7626 (1990).

${ }^{11}$ V. A. Shchukin and K. B. Efetov, Phys. Rev. B 43, 14164
(1991); J. Wang and J. P. Leburton, ibid. 41, 7846 (1990).

${ }^{12}$ B. Y.-K. Hu and S. Das Sarma, Phys. Rev. Lett. 68, 1750 (1992).

${ }^{13}$ H. Sakaki, Jpn. J. Appl. Phys. 19, L735 (1980).

${ }^{14}$ N. C. Constantinou et al., J. Phys. Condens. Matter 4, 4499 (1992); M. Masale et al., Phys. Rev. B 46, 15432 (1992).

${ }^{15}$ S. V. Branis et al., Phys. Rev. B 47, 1316 (1993); M. E. Rensink, Am. J. Phys. 37, 900 (1969).

${ }^{16}$ P. F. Williams and A. N. Bloch, Phys. Rev. B 10, 1097 (1974).

${ }^{17}$ W. I. Friesen and B. Bergersen, J. Phys. C 13, 6627 (1980).

${ }^{18}$ R. B. Dingle, Proc. R. Soc. London Ser. A 212, 47 (1952).

${ }^{19}$ K. S. Singwi et al., Phys. Rev. 176, 589 (1968).

${ }^{20}$ N. C. Constantinou and B. K. Ridley, J. Phys. Condens. Matter 1, 2283 (1989). 